\title{
ANÁLISE TEMPORAL DA COBERTURA FLORESTAL E USO DA TERRA NA APA DE GUARATUBA - PR
}

\author{
COVER TEMPORAL ANALISYS IN THE GUARATUBA ENVIRONMENTAL \\ PROTECTION AREA - PR \\ Jaqueline de Paula Heimann ${ }^{1}$, Francelino Sczanoski de Jesus Júnior ${ }^{2}$ \\ 1. Universidade Federal do Paraná, Curitiba, PR, Brasil - jaquelineheimann@yahoo.com.br \& ${ }^{2} S T C P$ \\ Engenharia de Projetos LTDA, Curitiba, PR, Brasil - linosjr@gmail.com
}

RESUMO

O crescimento e ocupação desordenada das terras contribuem para a perda de diversidade biológica nos biomas brasileiros. A criação de áreas especialmente protegidas, como as Áreas de Proteção Ambiental, unidades de conservação da categoria uso sustentável, consiste em um instrumento eficiente para frear tal cenário. Nesse sentido, a APA de Guaratuba, no estado do Paraná, desempenha importante papel de proteção da biodiversidade e controle da ocupação deste espaço, no entanto, é essencial que existam mecanismos de avaliação periódica do seu estado de conservação, por meio da identificação de ameaças que possam surgir à área protegida. Deste modo, o presente estudo objetivou quantificar a dinâmica da ocupação da terra na Área de Proteção Ambiental de Guaratuba - PR. Mapas temáticos de classificação da cobertura da terra foram elaborados para os anos de 1992 e 2017, no intuito de analisar as alterações ocorridas na área de estudo neste mesmo período. Foram utilizadas imagens a partir do TM Landsat, realizou-se a correção atmosférica das imagens com base no método Dark Object Subtraction (DOS). O sistema de projeção adotado foi o Universal Transversa de Mercator - UTM, fuso 22 Sul, Datum WGS-84. Foram definidas as classes de uso e iniciou-se o processo de classificação das imagens, empregando o Software ArcGis para a classificação supervisionada, a partir do algoritmo Maximum Likelihood - Maxver. Os resultados mostram que, ao longo dos 25 anos as áreas ocupadas por floresta nativa, agricultura e áreas consolidadas aumentaram, ao passo que as áreas ocupadas com água, campos, mangue e reflorestamentos diminuíram. Os coeficientes Kappa determinados para as classificações tanto de 1992 quanto 2017 apresentaram qualidade "muito boa" ou "concordância substancial", confirmando a acurácia da amostragem. Conclui-se que as principais modificações ocorreram nas classes agricultura e reflorestamento, havendo o aumento das áreas utilizadas para agricultura $(0,60 \%)$ e diminuição dos reflorestamentos $(-0,61 \%)$.

PALAVRAS-CHAVE: Sensoriamento remoto, Unidade de conservação, Uso e ocupação da terra.

\begin{abstract}
The disorderly growth and occupation of land contribute to the loss of biological diversity in Brazilian biomes. The creation of specially protected areas, such as Environmental Protection Areas, conservation units of the sustainable use category, is an efficient tool to stop such a scenario. In this sense, the Guaratuba APA, in the state of Paraná, plays an important role in protecting biodiversity and controlling the occupation of this space. However, it is essential to have mechanisms for periodic assessment of its conservation status, through the identification of threats. that may arise in the protected area. Thematic maps of land cover classification were prepared for the years 1992 and 2017, in order to analyze the changes in the study area during this same period, this study aimed to quantify the dynamics of land occupation in the Protected Area. Environmental Guaratuba - PR. The results show that over the 25 years the areas occupied by native forest, agriculture and consolidated areas increased, while the areas occupied by water, fields, mangroves and reforestation decreased. The Kappa coefficients determined for the 1992 and 2017 classifications presented "very good" or "substantial agreement" quality, confirming the accuracy of the sampling. It is concluded that the main changes occurred in the agriculture and reforestation classes, with an increase in the areas used for agriculture $(0.60 \%)$ and a decrease in reforestation $(-0.61 \%)$.
\end{abstract}

KEYWORDS: Remote sensing, Conservation unit, Land use and occupation. 


\section{INTRODUÇÃO}

A cobertura vegetal configura-se como um importante indicador das condições ambientais além de cooperar para manter a biodiversidade de uma região protegendo o solo, reduzindo o transporte de sedimentos e o assoreamento dos cursos de água e servindo de hábitat para animais silvestres (SAITO et al., 2016). Porém, os processos de fragmentação da vegetação e transformação das paisagens, cada vez mais comuns, intensificaram a pressão sobre as áreas naturais, resultando em ambientes pouco diversificados, com fragmentos florestais isolados e de dimensões reduzidas (CALEGARI et al., 2010).

A Mata Atlântica brasileira é considerada patrimônio nacional pela Constituição Federal de 1988, e sua utilização será feita conforme lei específica, dentro de condições que assegurem a preservação do ambiente, inclusive quanto ao uso dos recursos naturais (BRASIL, 1988). Assim, o bioma da Mata Atlântica possui $11,76 \%$ de sua cobertura original (RIBEIRO et al., 2009) e sua conservação, proteção, regeneração e utilização observam o que estabelece a Lei no 11.428 de 2006, conhecida como Lei da Mata Atlântica.

No ano de 1992, por meio do Decreto Estadual no 1.234, foi criada a Área de Proteção Ambiental - APA, denominada Guaratuba, abrangendo parte dos Municípios de Guaratuba, Matinhos, Tijucas do Sul, São José dos Pinhais e Morretes, com extensão de 199.596,5131 hectares e objetivo de compatibilizar o uso racional dos recursos ambientais da região com a ocupação ordenada do solo, protegendo a rede hídrica, os remanescentes de Floresta Atlântica e manguezais, os sítios arqueológicos e a diversidade faunística, disciplinando o uso turístico e garantindo a qualidade de vida das comunidades caiçaras e da população local (PARANÁ, 1992).

Entende-se por APA, as Unidades de Conservação UC's sob a tutela do Estado, com escopo de preservação de áreas florestais, ecossistemas de manguezais e bens culturais, tendo estas áreas, legislação disciplinar própria, e o principal objetivo de proteger os ecossistemas locais ao mesmo passo em que promove o desenvolvimento econômico e social.

Já o Sistema Nacional de Unidades de Conservação/SNUC classifica as Áreas de Proteção Ambiental como Unidades de Uso Sustentável, ou seja, diferentemente das demais modalidades de Unidades de Conservação, o uso econômico direto dos recursos naturais é disciplinado, não proibido e até incentivado (FERREIRA et al., 2009).

Diante da importância e fragilidade da APA de Guaratuba, torna-se importante conhecer a cobertura vegetal, bem como a estrutura da paisagem para auxiliar no monitoramento e planejamento do ambiente. Para isso, o emprego de dados de sensoriamento remoto, como por exemplo, as imagens orbitais, para detectar, mapear e quantificar os alvos da superfície terrestre são essenciais.

Saito et al. (2016), com o intuito de auxiliar na compreensão da complexidade de uma paisagem e sua influência nas relações ecológicas e na estrutura da paisagem, propõem a utilização de métricas de ecologia da paisagem como dado complementar aos estudos de interpretação de imagens.

Nesse sentido, a utilização de métricas, associadas às ferramentas computacionais para extrair informações de imagens, como a mineração de dados espaciais e espectrais, podem ser utilizada com intuito de potencializar a análise e aplicações de dados de sensoriamento remoto, tornando-se ferramentas importantes para tomadas de decisões (SAITO et al., 2016).

O uso de imagens de satélite, como fonte primária para elaboração de mapeamento, tem estimulado a área da geotecnologia, tendo em vista a variedade temporal de imagens disponíveis, e associadas ao baixo custo, quando o objetivo é obter o uso e cobertura do solo (DEMARCHI et al., 2011).

Diante do exposto, o estudo objetivou utilizar técnicas de classificação tradicional de imagens de satélite e quantificação de diferentes tipos de cobertura da terra presentes em uma análise multitemporal de imagens, para tanto utilizou-se imagens dos anos de 1992 e 2017, na APA de Guaratuba, Paraná.

\section{MATERIAL E MÉTODOS}

\section{Área de estudo}

A APA de Guaratuba localiza-se no litoral do estado do Paraná, ao sul do Brasil. Em sua extensão é possível encontrar as duas grandes unidades de relevo Paranaense, a Planície Litorânea ou Costeira e a Serra do Mar. O clima da região da APA de Guaratuba é caracterizado como Cfa, subtropical úmido (classificação de Köeppen), com altitude variando entre 280 e $550 \mathrm{~m}$ (CUNHA, 2010).

Ocorrem na APA de Guaratuba distintas formações vegetais: desde manguezais, que são formações especializadas ao meio e com pouca diversidade, até florestas mais complexas, ricas em espécies e formas de vida (FERNANDES \& BANDEIRA, 2014). Também estão inseridas no perímetro da APA de Guaratuba, o Parque Estadual do Boguaçu, com 6.052ha, o Parque Nacional Saint Hilaire/Lange, com 25.161ha e a Lagoa do Parado, 
considerada de utilidade pública (KANTEK, et al., 2009).

A APA possui qualidades ambientais relevantes à conservação e apresenta certa ocupação humana, pelo fato de possuir tanto terras de domínio público como privado. Existe uma série de restrições quanto ao uso da terra e dos recursos naturais com o objetivo de disciplinar o extrativismo por parte das comunidades existentes. De forma ampla, a área possui atributos bióticos e abióticos, estéticos ou culturais importantes para a qualidade de vida (KANTEK et al., 2009).

De acordo com o Plano de Manejo da APA de Guaratuba, por se tratar de UC da categoria Uso Sustentável, é essencial que atividades econômicas rentáveis, como os plantios de Pinus sp., sejam mantidas, desde que respeitem a legislação ambiental vigente e a regulamentação do zoneamento da APA. Na data da publicação do Plano de Manejo, 2006, a área total de reflorestamentos de Pinus era de aproximadamente 2.559 hectares o que corresponde a 1,29\% do total do uso da terra (PARANÁ, 2006).

Assim, em conjunto com as APAs de Guaraqueçaba e da Serra do Mar, a APA de Guaratuba conclui a mais completa Unidade de Conservação da Floresta Atlântica dentro de um Estado (KANTEK et al., 2009).

\section{Métodos}

As bases cartográficas referentes aos limites das unidades de conservação, municípios, vias e vias de acesso, necessárias para realizar as análises em questão foram obtidas gratuitamente do portal de mapas do Ministério do Meio Ambiente - MMA.

Por meio do avanço tecnológico e a facilidade na aquisição das informações da superfície, provenientes dos sensores orbitais, as imagens de satélite tornaram-se uma alternativa de grande relevância para o planejamento, seja ele em atividades de campo, ou até coleta de dados de maneira remota (ANDRADE, et al., 2014). Dessa forma para realizar as análises da evolução do uso da terra, foram utilizadas imagens de satélite de dois períodos distintos 1992 e 2017.

As imagens escolhidas para o trabalho foram as da família Landsat. Para o período 1992 as análises foram realizadas a partir do TM Landsat 5, de 19 de dezembro de 1992. Já para o período de 2017, utilizou-se o Landsat 8 OLI de 2 de agosto de 2017, ambas as datas com órbita ponto 220/78 objetivando abranger toda a área de estudo. A imagens foram obtidas gratuitamente através do United StatesGeologicalSurvey - USGS.

As imagens do Landsat apresentam bandas que exploram as regiões do visível, infravermelho próximo, médio e distante, permitindo montar composições coloridas que favoreçam a interpretação da cobertura vegetal e uso da terra devido suas respostas espectrais. Para o processamento inicial, realizou-se a correção atmosférica das imagens com base no método Dark Object Subtraction (DOS) proposto por Chavez (1988). Nesse método interferência atmosférica é estimada por Números Digitais-ND na imagem de satélite, situação que ignora a absorção atmosférica (WEISS, 2015). A aplicação desse método deu-se no software ArcGis 10, por meio de um complemento chamado de Geobia, que utiliza os paramentos nos metadados das imagens para as correções atmosféricas.

O sistema de projeção adotado foi o Universal Transversa de Mercator - UTM, fuso 22 Sul, Datum WGS84. No Landsat 5 , a combinação das bandas foram a $5(R)$, 4(G) e 3(B), que apresenta claramente os limites entre solo, água e vegetação (POLIDORO et al., 2009). Para a composição do satélite Landsat 8 , visando obter as mesmas respostas espectrais e padrão de cor, as bandas utilizadas foram a 6(R), 5(G) e 4(B).

Após a composição das imagens coloridas, foram definidas sete classes de uso da terra: área consolidada, agricultura, reflorestamento, floresta nativa, mangue, campos e corpos d'água, assim como definido no Manual de Uso da Terra, do Instituto Brasileiro de Geografia e Estatística - IBGE de 2013. Segundo esta fonte, a construção de uma nomenclatura do uso e cobertura da terra necessita estar adequada para mapear a diversidade do território considerado. Dessa forma, as classes observadas no manual de uso da terra, foram adaptadas para a área deste estudo.

Com as classes de uso definidas, iniciou-se o processo de classificação das imagens para os dois períodos, empregando a metodologia descrita por Nascimento et al. (2016), que utiliza o Software ArcGis para a classificação supervisionada, a partir do algoritmo Maximum Likelihood - Maxver. Esse algoritmo usa a informação espectral de maneira isolada para cada pixel da imagem e encontra regiões semelhantes e homogêneas (DEMARCHI et al., 2011).

Para executar o algoritmo Maxver, foi utilizada a ferramenta Image Classification do modulo Spatial Analyst (ArcGis), realizando um treinamento orientado ao objeto nas imagens, selecionando nas imagens 30 amostras para cada classe temática pré-definida, conforme proposto por Nascimento et al., (2016) (Figura 1). 


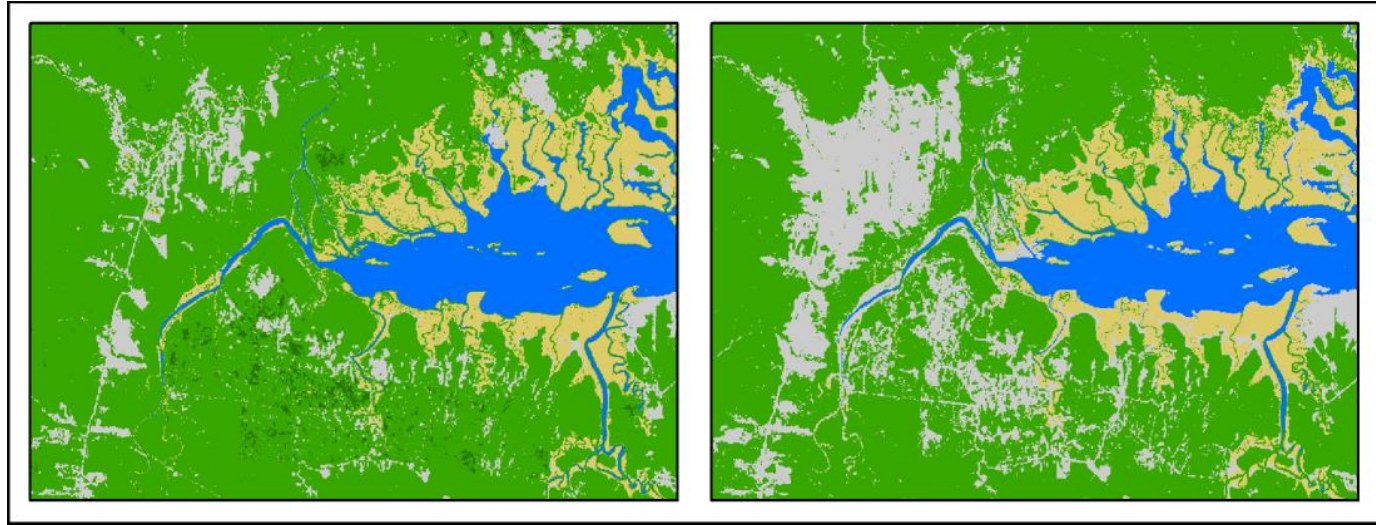

Figura 1 - A- Classificação do Uso da Terra Preliminar de 1992 / B-Classificação do Uso da Terra Preliminar de 2017.

No resultado da classificação supervisionada preliminar foram verificados ruídos de interpretação em de até 5 pixels agregados, dessa forma, foi aplicado um filtro de generalização que removeu agrupamentos de até 5 pixels (ou 0,45 ha) de ruídos nas imagens. Esse processo foi realizado por meio das ferramentas Region Group, Niblle e Set Null, disponíveis no módulo Spatial Analyst, Tools (ArcGis). Tais ferramentas, quando combinada, substituem as células de um raster correspondente a uma máscara com os valores dos vizinhos mais próximos.

Posteriormente, as classificações, 1992/2017 foram convertidas em um formato vetorial (shapefile) e recortadas no limite da APA de Guaratuba, com o intuito de realizar uma edição final de caráter visual, principalmente nas áreas com incidência de nuvens, bem como quantificar a evolução das classes de uso da terra.

Para uma validação da classificação via sensoriamento remoto, utilizou-se o cálculo do índice de concordância Kappa, que avalia a exatidão geral e por categoria do mapeamento e que, segundo Rezende et al. (2011) possibilita a identificação dos erros de omissão e comissão entre a classificação do mapa temático produzido e a verdade terrestre por meio de uma matriz.

Em seguida criou-se um arquivo vetorial de polígonos amostrais por meio do módulo Image Classification, que foi sobreposto sobre a respectiva composição colorida falsacor 543, não recortada, para anotação do indicador da classe correta de uso da terra em um arquivo de formato avl. Em seguida, por intermédio do módulo Assign, os indicadores corretos de uso da terra foram associados ao arquivo vetorial de pontos de amostragem, para então gerar o coeficiente Kappa, cruzando-se o arquivo gerado no módulo Assign com a imagem classificada. Após obter os padrões das amostras classificadas foi organizada uma tabela para aplicação do índice Kappa, por meio da seguinte equação:

$$
K=P_{0}-P_{e} / 1-P_{e}
$$

$\mathrm{PO}=$ Referente a exatidão da classificação (soma da coluna diagonal da matriz dividida pelo número total de "pixels" amostrados); $\mathrm{Pe}=\Sigma \mathrm{pi}+\mathrm{p}+\mathrm{i}$. $\mathrm{pi}+\mathrm{e} \mathrm{p}+\mathrm{i}$ as proporções marginais da linha " $\mathrm{i}$ " e da coluna " $\mathrm{i}$ ", respectivamente.

Dois coeficientes diferentes foram calculados, um para cada imagem classificada, considerando os dois anos de estudo, 1992 e 2017. Essa medida de concordância varia de < 0 (nenhuma concordância) a 1 (concordância total). A seguir são apresentadas as classificações do Índice de Kappa segundo Borges \& Sano (2014) e Demarchi et al. (2011) (Tabela 1).

Tabela 1. Qualidade da classificação associada aos valores do Índice Kappa.

\begin{tabular}{c|c}
\hline Borges \& Sano (2014) & Demarchi et al. (2011) \\
\hline$<0=$ sem concordância & $<0=$ péssima \\
$0-0,19=$ concordância pobre & $0-0,20=$ ruim \\
$0,20-0,39=$ concordância razoável & $0,20-0,40=$ razoável \\
$0,40-0,59=$ concordância \\
$\begin{array}{c}\text { moderada } \\
0,60-0,79=\text { concordância } \\
\text { substancial }\end{array}$ & $0,40-0,60=$ boa \\
$0,60-0,80=$ muito \\
boa
\end{tabular}

Fonte: adaptado de Borges e Sano (2014) e Demarchi et al. (2011).

\section{RESULTADOS E DISCUSSÃO}

Á área total da APA de Guaratuba que consta no Decreto de criação é de 199.596,51 hectares, já a área total da base cartográfica disponibilizada pelo Ministério do Meio Ambiente é de 199.445,27 hectares, ou seja, há uma diferença de 151,24 hectares para menos. O metadado da base cartográfica da UC, que é o arquivo que mostra os parâmetros na construção geral da base, informa que o 
limite da unidade de conservação foi construído a partir do memorial descritivo do decreto de criação da APA de 1992, entretanto, a UC faz limite com diversos rios e córregos, que podem justificar essa diferença, devido a escala de interpretação dos rios quando o limite foi construído.

Com base nas classificações a análise comparativa temporal entre as classes de uso da terra no período é possível constatar algumas alterações em sua configuração. No período considerado houve um aumento significativo na área de agricultura, equivalente a 1.192,35ha ou 0,6\% da área total. A diminuição da área de reflorestamento foi quase equivalente ao aumento da agricultura, ou seja, 1.206,97ha a menos de plantios florestais, ou $-0,61 \%$ da área total da UC.

A Tabela 2 apresenta as classes de uso da terra da Área de Proteção Ambiental de Guaratuba - PR.

Tabela 2. Área das classes de uso da terra da APA de Guaratuba em 1992 e 2017.

\begin{tabular}{ccccc}
\hline Classes de Uso & Uso 1992 & Uso 2017 & Diferença & $\%$ \\
\hline Agricultura & $5.532,45$ & $6.724,80$ & $1.192,35$ & 0,60 \\
\hline Água & $5.024,76$ & $4.833,40$ & $-191,36$ & $-0,10$ \\
Área Antropizada & $11.712,70$ & $12.048,84$ & 336,14 & 0,17 \\
\hline Campos & $3.470,76$ & $2.938,78$ & $-531,98$ & $-0,27$ \\
\hline Floresta Nativa & $161.929,65$ & $162.620,48$ & 690,82 & 0,35 \\
\hline Mangue & $6.990,52$ & $6.701,52$ & $-289,00$ & $-0,14$ \\
\hline Reflorestamento & $4.784,42$ & $3.577,45$ & $-1.206,97$ & $-0,61$ \\
\hline Total & $199.445,27$ & $199.445,27$ & 0,00 & 0,00 \\
\hline
\end{tabular}

As Figuras 4 e 5, além de apresentarem de forma ilustrativa as classes de uso mostradas na Tabela 1, ainda apresentam a área ocupada por cada classe, obtidas a partir da classificação supervisionada por Maxver das imagens Landsat-5 TM, nos anos de 1992 e 2017.

Ocorreu o aumento da área de floresta nativa em 690,82 hectares, ou $0,35 \%$ ao passo que a área ocupada por campos diminuiu em 531,98 hectares, equivalente a $0,27 \%$ da área total. Em menor proporção diminuiu a área ocupada por mangue, um total de 289 hectares a menos, ou $-0,14 \%$ da área da APA.

A menor variação foi percebida na área ocupada por corpo d'água, redução equivalente a 191,36 hectares, ou seja, $-0,1 \%$ da área total estudada.

Ainda se destaca o aumento da área antropizada, que em 1992, ano de criação da APA, era equivalente a $11.712,70$ hectares, passando a $12.048,84$ hectares em 2017 , aumento de $0,17 \%$ ou 336,14 hectares.
A análise das figuras mostra que a região central da APA, onde havia em 1992 predomínio de agricultura, se expandiu em 2017 sobre áreas que eram ocupadas por florestas nativas. Na mesma região é possível notar aumento da antropização, que decorre justamente na expansão da agricultura.

Na porção Sudoeste da APA é possível notar que em 1992 havia grande área ocupada por campos e por reflorestamento, especialmente de Pinus sp., e que ambas as áreas diminuíram em 2017, cedendo espaço à regeneração da floresta nativa.

Na porção Sudeste da APA, próximo ao Parque Estadual do Bogaçu, também era possível identificar em 1992 fragmentos de reflorestamento, o que não se vislumbra em 2017, onde houve antropização e diminuição da área ocupada por mangue. Vale ressaltar que os mangues são considerados Área de Preservação Permanente - APP pela Lei Florestal, Lei 12.651/12, ainda assim, segundo Madi et al. (2016) são áreas de intensas atividades antrópicas. Silva et al. (2015) ao estudarem a ocupação antrópica em manguezais de municípios litorâneos paranaenses concluíram que o crescimento urbano populacional vem ocorrendo e expandindo-se horizontalmente sobre os manguezais, de forma irregular e desordenada, em afronta à legislação ambiental. Não é possível identificar visualmente pelas Figuras em que porção da APA houve diminuição da área ocupada por corpos d'água. A redução da área de corpos d'água pode na verdade ser um efeito do aumento da área de nativas, especialmente de APP de beira de rio, mata ciliar, que escode os corpos d'água. Sugere-se que novos estudos sejam realizados com amostragem em campo para validar essa teoria.

Quanto à exatidão da classificação das imagens geradas, os coeficientes Kappa determinados para as classificações de 1992 e 2017 mostraram: para a classificação de 1992 o coeficiente Kappa foi de 0,72, qualidade "concordância substancial" ou "muito boa" segundo valores definidos na Tabela 1; o coeficiente Kappa da imagem classificada de 2017 apresentou valor de 0,66. Em que pese os valores serem pouco menores em comparação ao ano de 1992, ainda assim a qualidade enquadrou-se em "concordância substancial" ou "muito boa" segundo Tabela 1, confirmando a acurácia da amostragem.

A análise temporal da dinâmica de ocupação e uso da APA de Guaratuba, efetuada contínua e periodicamente, poderá ser utilizada como ferramenta para o monitoramento da cobertura da terra, detectando possíveis degradações ambientais que possam comprometer a sua integridade. 


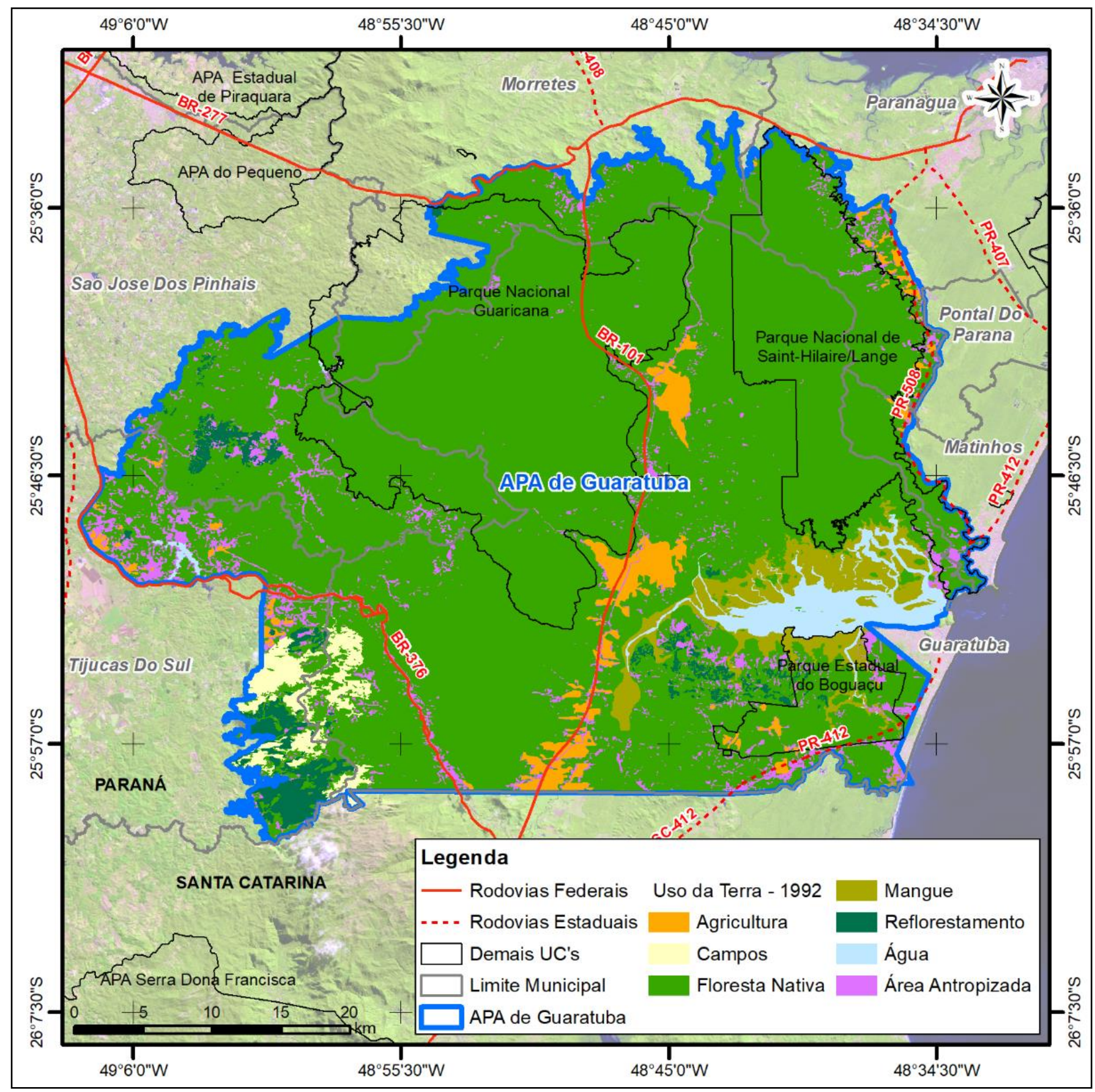

Figura 2. Classificação do Uso da Terra da Área de Proteção Ambiental de Guaratuba no ano de 1992. 


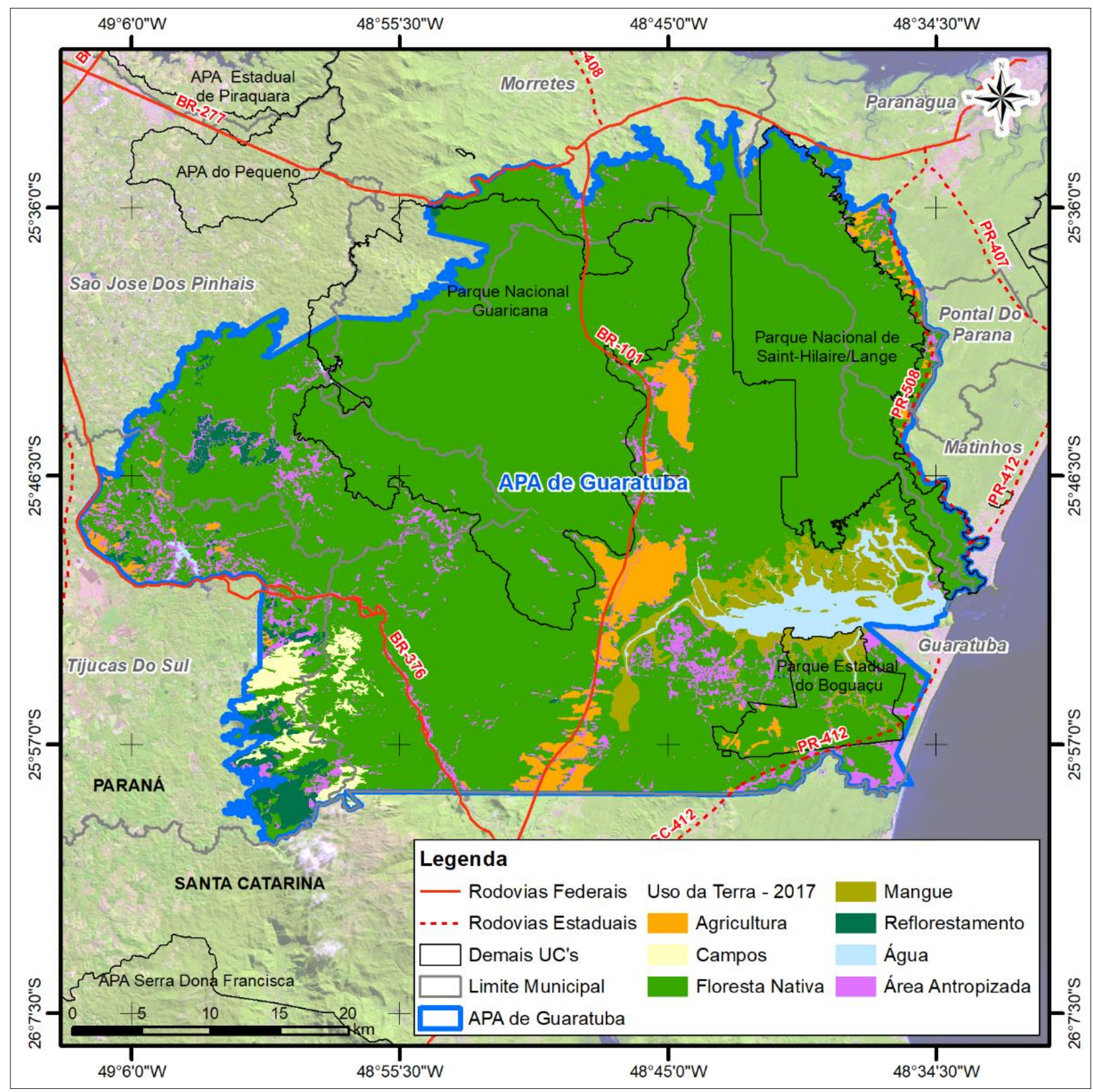

Figura 3. Classificação do Uso da Terra da Área de Proteção Ambiental de Guaratuba no ano de 2017.

\section{CONCLUSÕES}

A área que abriga a APA de Guaratuba teve acréscimo no tamanho total ocupado por floresta nativa, desde a sua criação em 1992. A floresta nativa ganhou espaço onde anteriormente havia reflorestamento e perdeu para agricultura e áreas consolidadas. Logo, não são os plantios de espécies exóticas, como Pinus sp., que pressionam a regeneração da Mata Atlântica no interior da APA de Guaratuba.

Logo, a orientação do Plano de Manejo de que atividades econômicas rentáveis, como os plantios de Pinus sp., sejam mantidas, desde que de acordo com as normas legais ambientais vigentes e a regulamentação do zoneamento da APA, não está sendo atendida.

Os resultados do coeficiente Kappa para os dois anos estudados, 1992 e 2017, atestam que, de maneira geral, a classificação da imagem de 1992 apresentou melhor exatidão no mapeamento, seguida da classificação da imagem de 2017, porém ambas classificadas como "muito boas" ou "concordância substancial" no que diz respeito a acurácia da classificação. 
A classificação de imagens do sensor Landsat-5 TM e Landsat-8OLI, com o propósito de monitorar a dinâmica da UC, mostrou-se eficiente e de uso relativamente acessível. A análise da dinâmica da APA pode ser indicada como instrumento auxiliar no gerenciamento da UC anualmente, como programas implantados na Amazônia Brasileira.

\section{AGRADECIMENTOS}

Os autores agradecem ao Centro de Aperfeiçoamento de Pessoal de Nível Superior (CAPES) pela concessão de bolsa de estudos de pós-graduação em Engenharia Florestal.

\section{REFERÊNCIAS}

ANDRADE, A.C. et al. Desempenho Dd classificadores paramétrico e não paramétrico na classificação da fisionomia vegetal. Revista Brasileira de Cartografia, n.66/2, p349-363, 2014.

BORGES, E.F.; SANO, E.E. Séries temporais de EVI do MODIS para o mapeamento de uso e cobertura vegetal do Oeste da Bahia. Boletim de Ciências Geodésicas, v.20, n.3, p.526-547, 2014.

BRASIL. Constituição da República Federativa do Brasil de 1988. Diário Oficial da União, Brasília, DF, cinco de outubro de 1988.

CALEGARI, L. et al. Análise da dinâmica de fragmentos florestais no município de Carandaí, MG, para fins de restauração florestal. Árvore, v.34, n.5, p.871-880, 2010.

CUNHA, A.K. et al. Anurofauna da Colônia Castelhanos, na Área de Proteção Ambiental de Guaratuba, Serra do Mar paranaense, Brasil. Biotemas, v.23, n.2, p.123-134, 2010.

DEMARCHI, J.C. et al. Análise Temporal do Uso do Solo e Comparação Entre os Índices de Vegetação NDVI e SAVI no Município de Santa Cruz do Rio Pardo - SP Usando Imagens Landsat-5. Revista RA'EGA, n.21, p.234-271, 2011.

FERNANDES, R.P.; BANDEIRA, D.R. Potencialidades da musealização de sítios arqueológicos: caso da APA de GuaratubaPR. Museologia e Patrimônio, v.7, n.1, p.77-94, 2014.

FERREIRA, M.R. et al. Comunidades rurais extrativistas da Mata Atlântica: uma abordagem sobre as famílias em Área de Proteção Ambiental no litoral paranaense. Curitiba: Editora UFPR, 2009.

IBGE - INSTITUTO BRASILEIRO DE GEOGRAFIA E ESTATÍSTICA. Manuais técnicos em geociência-manual técnico de uso da terra. 3.ed. Rio de Janeiro, 2013.

KANTEK, R.T. et al. Impactos ambientais na Área de Proteção Ambiental (APA) de Guaratuba, Paraná, Brasil, sob o ponto de vista de moradores tradicionais. Sociedade e Natureza, v.21, n.2, p.39-56, 2009.

MADI, A.P.L.M. et al. Estrutura do componente de regeneração natural e arbóreo de dois manguezais no estado do Paraná. Ciência Florestal, v.26, n.1, p.159-170, 2016.
NASCIMENTO, I.S. et al. Avaliação da exatidão dos classificadores Maxver e ISO Cluster do software Arcgis for desktop, com uso de imagem Landsat 8 do município de Cáceres/MT. Revista Continentes, v.5, n.8, p.48-62, 2016.

PARANÁ. Decreto Estadual n. 1.234 de 27 de março de 1992. Curitiba, 1992.

PARANÁ. PLANO de MANEJo dA ÁREA DE PROTEÇÃo AMBIENTAL DE GUARATUBA. Secretaria de Estado do Meio Ambiente E Recursos Hídricos (SEMA), Instituto Ambiental do Paraná (IAP), Programa Proteção da Mata Atlântica (PRÓATLÂNTICA). Curitiba, 2006.

POLIDORO, M. et al. Análise temporal do processo de conurbação na região de Londrina-PR por meio de imagens Landsat. Revista Geográfica Acadêmica, v.3, n.1, p.70-77, 2009.

REZENDE, R.A. et al. Análise temporal da flora nativa no entorno de Unidades de Conservação - APA Cachoeira das Andorinhas e Floe Uaimii, Ouro Preto, MG. Árvore, v.35, n.3, p.435-443, 2011.

RIBEIRO, M.C. et al. The Brazilian Atlantic Forest: How much is left, and how is the remaining forest distributed? Implications for conservation. Biological Conservation, v.142, p.1141-1153, 2009.

SAITO, N.S. et al. Uso da geotecnologia para análise temporal da cobertura florestal. Cerne, v.22, n.1, p.11-18, 2016.

SILVA, C.E. et al. A expansão urbana sobre manguezais no município de Paranaguá: o caso dos bairros Jardim Iguaçu e Vila Marinho. Revista Nacional de Gerenciamento de Cidades, v.3, n.14, p.92-111, 2015.

WEISS, C.V. et al. Análise comparativa entre métodos de correção atmosférica em imagens do sensor Operational Land Imager (OLI), plataforma Landsat 8. Scientia Plena, v.11, n.2, p.021711, 2014.

\section{Recebido em 26-08-2020 Aceito em 06-10-2020}

